\title{
DIVERGENCE-FREE WAVELETS FOR COHERENT VORTEX EXTRACTION IN 3D HOMOGENEOUS ISOTROPIC TURBULENCE
}

\author{
Erhan Deriaz ${ }^{1}$, Margarete O. Domingues ${ }^{2,3,4}$, Valérie Perrier ${ }^{1}$, \\ KAI SCHNEIDER ${ }^{3}$ AND MARIE FARGE ${ }^{4}$
}

\begin{abstract}
In this paper we investigate the use of divergence-free wavelet bases for the Coherent Vortex Extraction (CVE) of turbulent flows. We begin with a short presentation of the construction of 3D divergence-free biorthogonal wavelets. We apply the CVE decomposition to a homogeneous isotropic turbulent flow, computed by a Direct Numerical Simulation (DNS) at resolution $240^{3}$ and upsampled to $N=256^{3}$. First, the CVE is applied to the vorticity field. Using the divergence-free wavelets for the vorticity field makes sense since the vorticity also verifies an incompressibility condition when the velocity does. The coherent part of the vorticity field is reconstructed from the largest wavelet coefficients, corresponding to $3 \% N$, while the complement constitutes the incoherent part. We show that the coherent part corresponds to the vortex tubes of the flow and retains most of the energy and enstrophy. These results are then compared to those obtained using non-divergent free wavelets, both orthogonal and biorthogonal ones. Then we also apply the CVE method, using divergence-free wavelets, to decompose the velocity field and subsequently compute the corresponding vorticity fields. The results show that the decomposition of velocity exhibit large smooth vortex structures in contrast to what is obtained with the decomposition of the vorticity.
\end{abstract}

Résumé. Dans cet article nous étudions l'utilisation des bases d'ondelettes à divergence nulle pour l'extraction de tourbillons cohérents (Coherent Vortex Extraction: CVE). Nous rappelons d'abord brièvement la construction des ondelettes à divergence nulle en dimension 3. Puis nous appliquons la méthode CVE pour la décomposition d'un champ turbulent homogène isotrope, issu d'une simulation directe de résolution $N=240^{3}$, et sur-échantillonné à $N=256^{3}$. Pour commencer, la décomposition CVE est appliquée au champ de vorticité. Utiliser les ondelettes à divergence nulle dans ce contexte a un sens, dans la mesure où si la vitesse vérifie une condition de divergence nulle, la vorticité la vérifie également. La partie cohérente du champ de vorticité est construite à partir des $3 \% N$ plus importants coefficients d'ondelettes, tandis que le complément constitue la partie incohérente de l'écoulement. Nous montrons que l'écoulement cohérent correspond aux tubes de vorticité et contient une grande part de l'énergie et de l'enstrophie du champ total. Ces résultats sont comparés à ceux obtenus avec des ondelettes orthogonales et biorthogonales, qui ne sont pas à divergence nulle. Ensuite nous appliquons les ondelettes à divergence nulle pour la décomposition CVE du champ de vitesse. Les visualisations des champs de vorticité correspondant montrent que, contrairement à ce qui était obtenu pour l'écoulement cohérent de la vorticité la partie cohérente de la vitesse met en évidence des tubes de vorticité réguliers.

\footnotetext{
1 Laboratoire de Modélisation et Calcul de l'IMAG, BP 53, 38041 Grenoble cedex 9, France.

${ }^{2}$ Laboratório Associado de Computação e Matemática Aplicada (LAC), Instituto Nacional de Pesquisas Espaciais (INPE), Av. dos Astronautas, 1758, 12227-010 São José dos Campos, Brazil.

3 MSNM-CNRS \& CMI, Université de Provence, 39 rue Joliot-Curie, 13453 Marseille cedex 13, France.

${ }^{4}$ LMD-CNRS, Ecole Normale Supérieure, 24 rue Lhomond, 75231 Paris cedex 05, France.

e-mail: Erwan.Deriaz@imag.fr \& margarete@lac.inpe.br \& Valerie.Perrier@imag.fr \&

kschneid@cmi.univ-mrs.fr \& farge@lmd.ens.fr
}

(C) EDP Sciences, SMAI 2007 


\section{Contents}

1. Introduction $r$

2. Divergence-free vector wavelets $r$

2.1. 3D wavelets in the scalar case $\quad 147$

2.2. Construction of div-free vector wavelets 148

3. Numerical results $\quad 151$

3.1. Principle of the CVE decomposition 151

3.2. DNS data 152

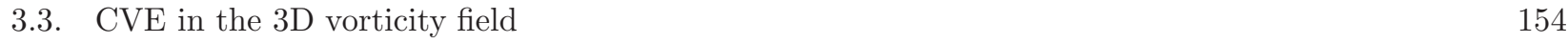

3.4. CVE in the 3D velocity field 156

4. Conclusion 157

$\begin{array}{lr}\text { References } & 157\end{array}$

\section{INTRODUCTION}

The Coherent Vortex Extraction (CVE) method has been introduced in different papers [9-11, 16]. The principle of the method consists in separating the flow into a coherent part and noise, which is supposed to be Gaussian and decorrelated. The CVE is based on a wavelet decomposition of the field (originally the vorticity field). A nonlinear approximation of the field, provided by the wavelet decomposition, and corresponding to the best- $\mathrm{N}$ term approximation, i.e., we retain the $N_{\mathrm{c}}$ largest wavelet coefficients in the wavelet expansion, $N_{\mathrm{c}}$ being chosen suitably. They correspond to the coherent part, whereas the remaining $97 \%$ of the coefficients represent the incoherent background flow.

In [15] the coherent vortex extraction has been studied to analyze a 3D homogeneous isotropic turbulent flow computed by Dinamical Numerical Simulations (DNS). In this paper we compare the CVE applied to the vorticity using, either divergent free biorthogonal wavelets, or orthogonal and biorthogonal non divergent free wavelets which have been presented in [15].

Both decompositions allow an efficient extraction of the coherent vortices retaining only few wavelet modes, i.e. $3 \% N$ of the coefficients. Divergence-free wavelets have been originally designed by Lemarié [13] and have been firstly used by Urban in the context of fluid mechanics, to analyze two-dimensional turbulent flows [1,19], as well as to compute the 2D/3D Stokes solution for the driven cavity problem [17].

A recent work of Deriaz and Perrier [6] describe an efficient algorithm to compute a divergence-free wavelet decomposition of any incompressible $2 \mathrm{D} / 3 \mathrm{D}$ vector field, and a way to compute the Leray-projection, i.e. the divergence-free part of any compressible field, directly in wavelet space.

Since for 3D incompressible flows, the velocity and vorticity fields are divergence-free, the coherent vortex extraction in the divergence free wavelet decomposition is applied to both fields. For both analyses, we will compare the coherent and incoherent parts of the flow with the total flow, and the corresponding statistics.

The paper is organized as follows: in section 2 , we recall the basics of $2 \mathrm{D} / 3 \mathrm{D}$ divergence-free wavelets. In section 3 we present results of the CVE applied to DNS data (vorticity and velocity) of 3D homogeneous isotropic turbulence. Finally, conclusions are given in section 4, where we present some perspectives for turbulence modeling.

\section{DivergenCE-FREE VECTOR WAVELETS}

\subsection{D wavelets in the scalar case}

Multivariate wavelet bases (orthogonal or biorthogonal) are obtained by tensor products of one-dimensional wavelets or scaling functions. The construction of one-dimensional wavelets is linked to Multiresolution Analyses 
(MRA), see e.g. [8,14]. In the following we will note by $V_{j}$ the multiresolution spaces, and $\phi$, $\psi$ the associated scaling functions and wavelets.

Isotropic wavelets versus anisotropic wavelets.

Isotropic wavelet bases are wavelet bases arising from the $3 \mathrm{D}$ MRA analyses $\mathcal{V}_{j}=V_{j}^{(1)} \otimes V_{j}^{(2)} \otimes V_{j}^{(3)}$ constructed by space tensor products. Here $V_{j}^{(i)}$ denotes a one-dimensional MRA, which can be different in each direction. In the standart setting the MRA are often identical in all directions, but it would not be the case in the divergence-free context. In such MRA, 3D scaling functions are given by:

$$
\Phi_{j, i_{x}, i_{y}, i_{z}}(\vec{x})=\phi_{j, i_{x}}^{(1)}(x) \phi_{j, i_{y}}^{(2)}(y) \phi_{j, i_{z}}^{(3)}(z)
$$

where $\phi_{j, k}^{(i)}(x)=2^{\frac{j}{2}} \phi^{(i)}\left(2^{j} x-k\right)$ are the $1 \mathrm{D}$ scaling functions of the MRA $V_{j}^{(i)}$ (when $k$ varies in $\mathbb{Z}$ ).

The corresponding $3 \mathrm{D}$ wavelets are

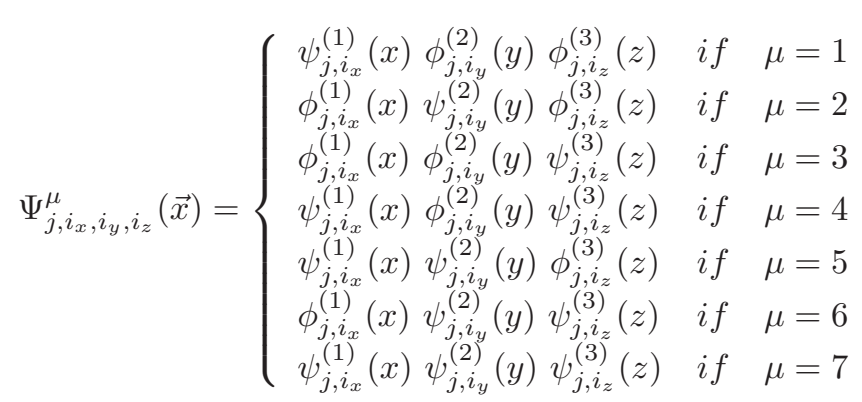

Notice that the corresponding support of each basis function is a cube of size $\sim 2^{-j}$, but these functions correspond to 7 different directions.

Anisotropic 3D wavelets are constructed by taking the tensor product of three 1D wavelet bases (which can be different) $\psi_{j, k}^{(i)}(x)=2^{j / 2} \psi^{(i)}\left(2^{j} x-k\right)$ (they are often called tensor-product wavelets). In this case, the basis functions are generated from "anisotropic" dilations of the following tensor product function:

$$
\Psi(x, y, z)=\psi^{(1)}(x) \psi^{(2)}(y) \psi^{(3)}(z)
$$

and they are given by:

$$
\Psi_{j_{x}, j_{y}, j_{z}, i_{x}, i_{y}, i_{z}}(\vec{x})=\psi_{j_{x}, i_{x}}^{(1)}(x) \psi_{j_{y}, i_{y}}^{(2)}(y) \psi_{j_{z}, i_{z}}^{(3)}(z)
$$

The above functions have different scales in different directions and thereforean anisotropic support.

\subsection{Construction of div-free vector wavelets}

Let

$$
\mathbf{H}_{\operatorname{div}, 0}\left(\mathbb{R}^{3}\right)=\left\{\mathbf{u} \in\left(L^{2}\left(\mathbb{R}^{3}\right)\right)^{3} ; \operatorname{div} \mathbf{u} \in L^{2}\left(\mathbb{R}^{n}\right), \quad \operatorname{div} \mathbf{u}=0\right\}
$$

be the space of divergence-free vector functions in $\mathbb{R}^{3}$.

Compactly supported divergence-free wavelets bases of $\mathbf{H}_{\text {div }, 0}\left(\mathbb{R}^{3}\right)$ were originally designed by P.G. LemariéRieusset, in the context of biorthogonal MRA [13], in the general case of $\mathbb{R}^{n}$. We describe here the principles of their construction, for more details on the related fast algorithms, we refer to [6].

\section{D divergence-free MRA}

The construction of divergence-free wavelet is based on the existence of two different one-dimensional multiresolution analyses of $L^{2}(\mathbb{R})$ related by differentiation and integration. Let $\left(V_{j}^{1}\right)_{j \in \mathbb{Z}}$ be a one-dimensional MRA, with a derivable scaling function $\phi_{1}$, (i.e. $V_{0}^{1}=\operatorname{span}\left\{\phi_{1}(x-k), k \in \mathbb{Z}\right\}$ ), and a wavelet $\psi_{1}$ : one can build a second MRA $\left(V_{j}^{0}\right)_{j \in \mathbb{Z}}$ with a scaling function $\phi_{0}\left(V_{0}^{0}=\operatorname{span}\left\{\phi_{0}(x-k), k \in \mathbb{Z}\right\}\right)$ and a wavelet $\psi_{0}$ verifying: 


$$
\phi_{1}^{\prime}(x)=\phi_{0}(x)-\phi_{0}(x-1) \quad \psi_{1}^{\prime}(x)=4 \psi_{0}(x) .
$$

An example of MRA satisfying equation (1) is given by splines of degree 1 ( $V_{j}^{0}$ MRA spaces) and splines of degree $2\left(V_{j}^{1}\right.$ MRA spaces). In both cases we draw the scaling functions $\phi_{0}, \phi_{1}$ and their associated wavelets $\psi_{0}, \psi_{1}$ with shortest support (Fig. 1).

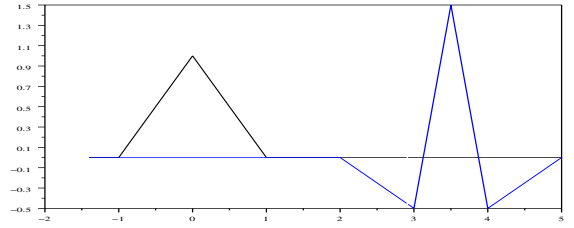

$\phi_{0}$

$\psi_{0}$

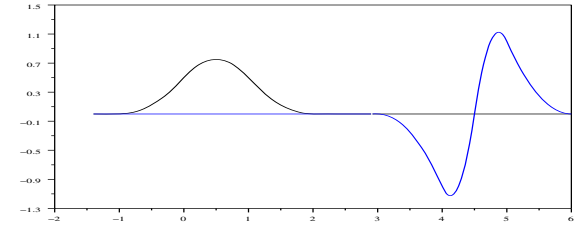

$\phi_{1}$

$\psi_{1}$

FIGURE 1. Scaling functions and associated even and odd wavelets with shortest support, for splines of degree 1 (left) and 2 (right).

To construct divergence-free scaling functions, we consider the following vector multiresolution analysis of $\left(L^{2}\left(\mathbb{R}^{3}\right)\right)^{3}$ :

$$
\left[\mathcal{V}_{j}=\left(V_{j}^{1} \otimes V_{j}^{0} \otimes V_{j}^{0}\right) \times\left(V_{j}^{0} \otimes V_{j}^{1} \otimes V_{j}^{0}\right) \times\left(V_{j}^{0} \otimes V_{j}^{0} \otimes V_{j}^{1}\right)\right]_{j \in \mathbb{Z}}
$$

The associated 3D vector scaling functions are given by:

$$
\begin{gathered}
\Phi_{1}(x, y, z)=\left|\begin{array}{l}
\phi_{1}(x) \phi_{0}(y) \phi_{0}(z) \\
0
\end{array} \quad \Phi_{2}(x, y, z)=\right| \begin{array}{l}
0 \\
\phi_{0}(x) \phi_{1}(y) \phi_{0}(z) \\
0
\end{array} \\
\Phi_{3}(x, y, z)=\mid \begin{array}{l}
0 \\
0 \\
\phi_{0}(x) \phi_{0}(y) \phi_{1}(z)
\end{array}
\end{gathered}
$$

From these scaling functions we can derive divergence free scaling functions:

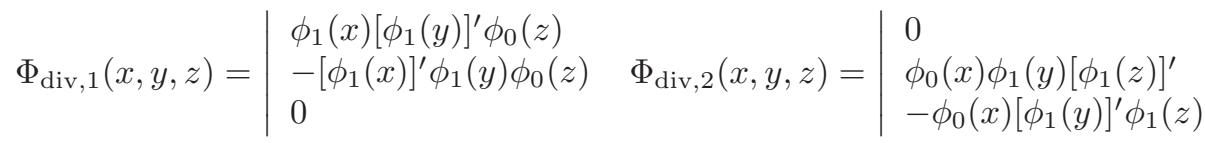

$$
\begin{aligned}
& \Phi_{\operatorname{div}, 3}(x, y, z)=\mid \begin{array}{l}
-\phi_{1}(x) \phi_{0}(y)\left[\phi_{1}(z)\right]^{\prime} \\
0 \\
{\left[\phi_{1}(x)\right]^{\prime} \phi_{0}(y) \phi_{1}(z)}
\end{array}
\end{aligned}
$$

which are linear combinations of the "standard" scaling functions, by using the relation $\phi_{1}^{\prime}(s)=\phi_{0}(s)-\phi_{0}(s-1)$ :

$$
\begin{aligned}
& \Phi_{\operatorname{div}, 1}(x, y, z)=\Phi_{1}(x, y, z)-\Phi_{1}(x, y-1, z)-\Phi_{2}(x, y, z)+\Phi_{2}(x-1, y, z) \\
& \Phi_{\operatorname{div}, 2}(x, y, z)=\Phi_{2}(x, y, z)-\Phi_{2}(x, y, z-1)-\Phi_{3}(x, y, z)+\Phi_{3}(x, y-1, z) \\
& \left.\Phi_{\operatorname{div}, 3}(x, y, z)=\Phi_{3}(x, y, z)-\Phi_{3}(x-1, y, z)-\Phi_{1}(x, y, z)+\Phi_{1}(x, y, z-1)\right)
\end{aligned}
$$


These functions generate a divergence-free MRA. As these three functions are linearly dependent $\left(\Phi_{\operatorname{div}, 1}+\right.$ $\left.\Phi_{\operatorname{div}, 2}+\Phi_{\operatorname{div}, 3}=0\right)$, we have to choose 2 scaling functions among the three above, for instance we can choose:

$$
\mathbb{V}_{\text {div }, 0}=\operatorname{span}\left\{\Phi_{\operatorname{div}, 1}(\vec{x}-\vec{k}) ; \Phi_{\operatorname{div}, 2}(\vec{x}-\vec{k}) ; \vec{k} \in \mathbb{Z}^{3}\right\}
$$

and define

$$
\mathbb{V}_{\operatorname{div}, j}=\operatorname{span}\left\{\mathbf{u}\left(2^{j} .\right) ; \mathbf{u} \in \mathbb{V}_{\operatorname{div}, 0}\right\}
$$

In this new divergence-free MRA, we can construct isotropic as well as anisotropic divergence free wavelet bases. In both cases the divergence-free wavelets are given by linear combinations of the "canonical" but vector valued wavelets of the MRA $\mathcal{V}_{j}$.

In the isotropic case, from the 21 canonical generating $3 D$ vector wavelets $\left\{\vec{\Psi}^{i, \mu} \mid i=1,2,3, \mu=1,7\right\}$ :

$$
\vec{\Psi}^{1, \mu}=\left|\begin{array}{ll}
\Psi^{\mu} \\
0 \\
0
\end{array} \quad \vec{\Psi}^{2, \mu}=\right| \begin{aligned}
& 0 \\
& \Psi^{\mu} \\
& 0
\end{aligned} \quad \vec{\Psi}^{3, \mu}=\mid \begin{aligned}
& 0 \\
& 0 \\
& \Psi^{\mu}
\end{aligned}
$$

one constructs 14 generating divergence-free wavelets $\Psi_{\text {div }}^{i, \mu},(i=1,2, \mu=1,7)$, and 7 complement functions $\Psi_{\mathrm{n}}^{\mu}$ $(\mu=1,7)$. Their exact forms can be found in [6]. We plot on Figure 2, an isosurface of the modulus of the vorticity field, associated to each divergence-free basis function.
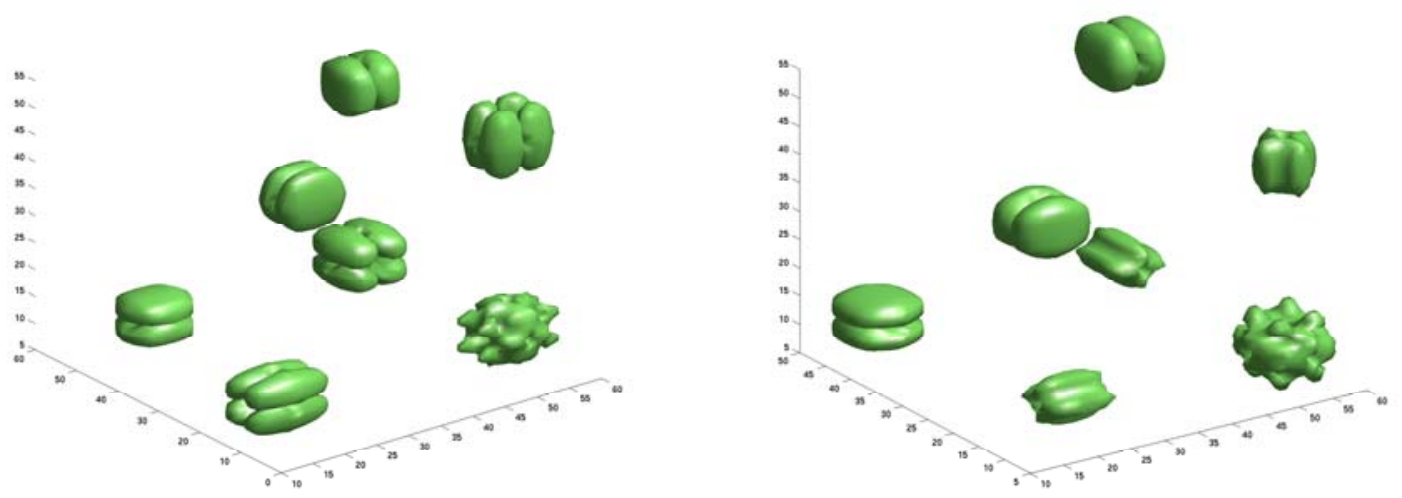

FiguRE 2. Isosurface of the modulus of the curl of the 14 div-free vector wavelets in $\mathbb{R}^{3}$.

Unlike the isotropic case, anisotropic divergence-free wavelets are generated from two vector functions:

$$
\Psi_{\operatorname{div}}^{\mathrm{an}, 1}(x, y, z)=\left|\begin{array}{l}
\psi_{1}(x) \psi_{0}(y) \psi_{0}(z) \\
-\psi_{0}(x) \psi_{1}(y) \psi_{0}(z) \\
0
\end{array} \quad \Psi_{\operatorname{div}}^{\mathrm{an}, 2}(x, y, z)=\right| \begin{aligned}
& 0 \\
& \psi_{0}(x) \psi_{1}(y) \psi_{0}(z) \\
& -\psi_{0}(x) \psi_{0}(y) \psi_{1}(z)
\end{aligned}
$$


by anisotropic dilations, and translations. Anisotropic three-dimensional divergence-free wavelets take the form:

$$
\begin{aligned}
& \Psi_{\text {div }, 1, \mathbf{j}, \mathbf{k}}^{\text {an }}\left(x_{1}, x_{2}, x_{3}\right)=\mid \begin{array}{l}
2^{j_{2}} \psi_{1}\left(2^{j_{1}} x_{1}-k_{1}\right) \psi_{0}\left(2^{j_{2}} x_{2}-k_{2}\right) \psi_{0}\left(2^{j_{3}} x_{3}-k_{3}\right) \\
-2^{j_{1}} \psi_{0}\left(2^{j_{1}} x_{1}-k_{1}\right) \psi_{1}\left(2^{j_{2}} x_{2}-k_{2}\right) \psi_{0}\left(2^{j_{3}} x_{3}-k_{3}\right) \\
0
\end{array} \\
& \Psi_{\text {div }, 2, \mathbf{j}, \mathbf{k}}^{\mathrm{an}}\left(x_{1}, x_{2}, x_{3}\right)=\mid \begin{array}{l}
0 \\
2^{j_{3}} \psi_{0}\left(2^{j_{1}} x_{1}-k_{1}\right) \psi_{1}\left(2^{j_{2}} x_{2}-k_{2}\right) \psi_{0}\left(2^{j_{3}} x_{3}-k_{3}\right) \\
-2^{j_{2}} \psi_{0}\left(2^{j_{1}} x_{1}-k_{1}\right) \psi_{0}\left(2^{j_{2}} x_{2}-k_{2}\right) \psi_{1}\left(2^{j_{3}} x_{3}-k_{3}\right)
\end{array}
\end{aligned}
$$

with $\mathbf{j}=\left(j_{1}, j_{2}, j_{3}\right), \mathbf{k}=\left(k_{1}, k_{2}, k_{3}\right) \in \mathbb{Z}^{3}$.

Decomposition of $\left(L^{2}\left(\mathbb{R}^{3}\right)\right)^{3}$ : Since divergence-free wavelets generate $\mathbf{H}_{\text {div }, 0}\left(\mathbb{R}^{3}\right)\left(\operatorname{and}\right.$ not $\left.\left(L^{2}\left(\mathbb{R}^{3}\right)\right)^{3}\right)$,

we have to introduce complement functions $\Psi_{\mathbf{n}, \mathbf{j}, \mathbf{k}}$ (see [6] for examples of such functions) to form a basis of the vector space $\left(L^{2}\left(\mathbb{R}^{3}\right)\right)^{3}$.

For instance in the isotropic case, it writes:

$$
\left(L^{2}\left(\mathbb{R}^{3}\right)\right)^{3}=\operatorname{span}\left\{\Psi_{\mathrm{div}, j, \mathbf{k}}^{i, \mu}\right\} \oplus \operatorname{span}\left\{\Psi_{\mathrm{n}, j, \mathbf{k}}^{\mu}\right\}
$$

The choice of these complement functions is not unique, and for a given compressible field, it induces the values of its divergence-free wavelet coefficients.

As divergence-free wavelets with compact support are not compatible with orthogonal MRAs, we can't find an orthogonal complement. Thus the decomposition is not orthogonal. However we have $\operatorname{rot} \Psi_{\mathrm{n}, j, \mathbf{k}}^{\mu} \neq 0$. Now we can write the wavelet decomposition of any vector field $\mathbf{u}$ :

$$
\mathbf{u}=\sum_{\mu, i, j, \mathbf{k}} d_{\operatorname{div}, j, \mathbf{k}}^{i, \mu} \Psi_{\mathrm{div}, j, \mathbf{k}}^{i, \mu}+\sum_{\mu, j, \mathbf{k}} d_{\mathrm{n}, j, \mathbf{k}}^{\mu} \Psi_{\mathrm{n}, j, \mathbf{k}}^{\mu}
$$

The computation of the coefficients $d_{\mathrm{div}, j, \mathbf{k}}^{i, \mu}$ corresponding to the div-free part is in pratice obtained through a standard wavelet decomposition of each component of $\mathbf{u}$, followed by a linear combination of these coefficients (see [6]). Then the complexity of a divergence-free wavelet decomposition is of the same order than a 3D Fast Wavelet Transform $(O(N)$ operations to compute $N$ coefficients).

Remark that if $\mathbf{u}$ is incompressible, the second term in the decomposition (3) vanishes, since we have:

$$
\operatorname{div} \mathbf{u}=\sum_{\mu, j, \mathbf{k}} d_{\mathrm{n}, j, \mathbf{k}}^{\mu} \operatorname{div} \psi_{\mathrm{n}, j, \mathbf{k}}^{\mu}=0
$$

where it can be seen that the family $\left(\operatorname{div} \psi_{\mathrm{n}, j, \mathbf{k}}^{\mu}\right)$ forms a Riesz basis of $L^{2}\left(\mathbb{R}^{3}\right)[6]$, applying $d_{\mathrm{n}, j, \mathbf{k}}^{\mu}=0$.

\section{Numerical RESUlts}

\subsection{Principle of the CVE decomposition}

We consider a 3D vector field, either velocity $\mathbf{u}$, or vorticity $\omega$. The principle of the coherent vortex extraction, in the divergence-free wavelet context, is as follows: First, the vector field $\mathbf{u}$ is developed into divergence-free vector wavelets and complement functions:

$$
\mathbf{u}=\sum_{\mu, i, j, \mathbf{k}} d_{\mathrm{div}, j, \mathbf{k}}^{i, \mu} \Psi_{\mathrm{div}, j, \mathbf{k}}^{i, \mu}+\sum_{\mu, j, \mathbf{k}} d_{\mathrm{n}, j, \mathbf{k}}^{\mu} \Psi_{\mathrm{n}, j, \mathbf{k}}^{\mu}
$$


Then a threshold is applied to the ( $L^{2}$-renormalized) wavelet coefficients, i.e., only wavelet coefficients whose modulus is large than a given threshold are retained in absolute value. In order to compare our results with those of [15], we choose a threshold $T$ such that the total number of coefficients retained in the coherent part corresponds to $3 \% N$ with $N=256^{3}$ here. The coherent part of the field is then:

$$
\mathbf{u}_{\mathrm{c}}=\sum_{\left|d_{\mathrm{div}, j, \mathbf{k}}^{i, \mu}\right|>T} d_{\operatorname{div}, j, \mathbf{k}}^{i, \mu} \Psi_{\operatorname{div}, j, \mathbf{k}}^{i, \mu}+\sum_{\left|d_{\mathbf{n}, j, \mathbf{k}}^{\mu}\right|>T} d_{\mathrm{n}, j, \mathbf{k}}^{\mu} \Psi_{\mathrm{n}, j, \mathbf{k}}^{\mu}
$$

Remark that if $\mathbf{u}$ is divergence free, the second term of the right hand side vanishes. But in practice, vector fields $\mathbf{u}$ arising from a spectral code will verify a div-free condition in the Fourier domain; after interpolation in the spline-wavelet domain, this divergence free condition is no more observed and one has to take into account the complement part.

The incoherent velocity is computed by the difference with the total field:

$$
\mathbf{u}_{\mathrm{i}}=\mathbf{u}-\mathbf{u}_{\mathrm{c}}
$$

Since the divergence-free wavelets and their complement functions form a biorthogonal (and not orthogonal) basis of $\left(L^{2}\left(\mathbb{R}^{3}\right)^{3}\right)$, the total energy verifies:

$$
E=\frac{1}{2}\left\|\mathbf{u}_{\mathrm{c}}+\mathbf{u}_{\mathrm{i}}\right\|^{2}=E_{\mathrm{c}}+E_{\mathrm{i}}+<\mathbf{u}_{\mathrm{c}} \mid \mathbf{u}_{\mathrm{i}}>
$$

In the same way, the CVE is applied to the vorticity field $\omega$, leading to a coherent vorticity $\omega_{\mathrm{c}}$ and an incoherent vorticity $\omega_{\mathrm{i}}=\omega-\omega_{\mathrm{c}}$. Similarly, the total enstrophy verifies

$$
Z=\frac{1}{2}\left\|\omega_{\mathrm{c}}+\omega_{\mathrm{i}}\right\|^{2}=Z_{\mathrm{c}}+Z_{\mathrm{i}}+<\omega_{\mathrm{c}} \mid \omega_{\mathrm{i}}>
$$

\subsection{DNS data}

We apply the CVE, with divergence-free wavelets, to the vorticity and velocity fields of a 3D homogeneous isotropic turbulent flow. The data are coming from a DNS (direct numerical simulation), using a pseudo-spectral code at resolution $240^{3}$ [20], upsampled to $256^{3}$. The flow is forced at the largest scale, and the turbulence level corresponds to a microscale Reynolds number $R_{\lambda}=150$, with

$$
R_{\lambda}=\frac{\lambda V_{r m s}}{\nu}
$$

and where $\lambda=(E / Z)^{1 / 2}$ denotes the Taylor microscale, $V_{r m s}$ the root-mean-square velocity, and $\nu$ the kinematic viscosity. Figure 6 shows a $64^{3}$ sub-cube of the modulus of vorticity what can we see vortex tubes.

The divergence free wavelets used in the numerical experiments are constructed from biorthogonal splines of degree 1 (spaces $V_{j}^{0}$ ) and 2 (spaces $V_{j}^{1}$ ) (see section 2 ). We begin with a comparison of the compression rates between isotropic and anisotropic wavelets, obtained through the nonlinear compression of the vorticity field.

Comparison of compression rates between isotropic and anisotropic div-free wavelets: Figure 3 represents the error provided by the nonlinear approximation, in terms of the number of retained coefficients (in semi-logarithmic scale).

As one can see on Figure 3, the compression curve (we represented the relative enstrophy of the incoherent part $\omega_{\mathrm{i}}$, versus the number of retained coefficients) associated to isotropic wavelets is already decreasing, whereas the one associated to anisotropic wavelets grows for a low number of retained coefficients (due to the non orthogonality), before decreasing. This comparison between isotropic and anisotropic divergence free wavelets clearly shows that for CVE purpose, the isotropic decomposition is more adapted, since we will highly compress the fields, by retaining a very few number of wavelet modes.

Compression rates of the divergence-free projection of the discrete vorticity field, and of its complement: Figure 4 represents the compression curves of the div-free part of the vorticity and of its complement 


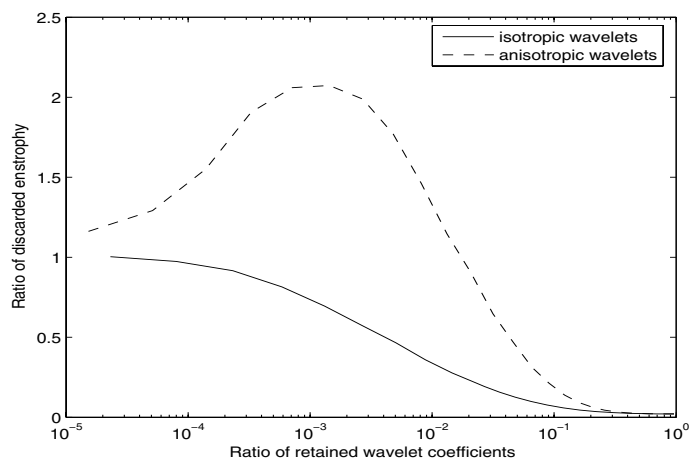

FIGURE 3. Comparison between isotropic (plain line) and anisotropic (dashed line) div-free wavelet compression of the vorticity field in semi-log scale.

part, when using isotropic wavelets (divergence-free wavelets and complement wavelets, as in decomposition $(2))$. As one can see, the complement part is not negligible, since the field we analyze does not verify a divergence free condition, after interpolation in the considered spline space. Nevertheless, the complement functions will represent less than $0.4 \%$ of the total coefficients retained in the $3 \%$-best terms approximation.

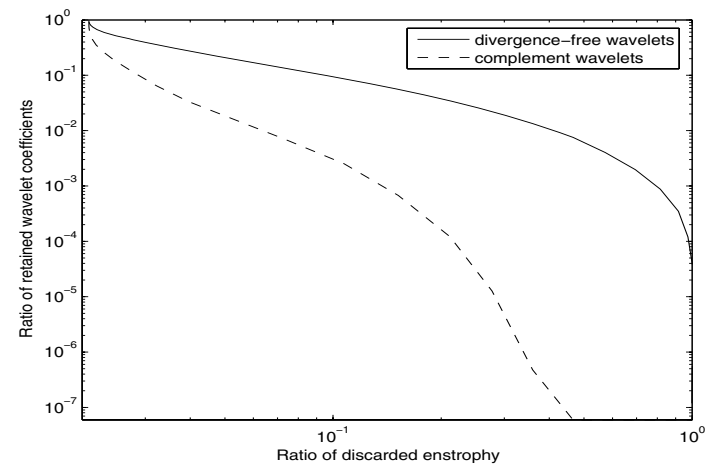

FIGURE 4. Compression error in terms of the number of retained coefficients (log-log scale): div-free part (plain line) and complement part (dashed line) of the vorticity field.

Compression rates of the divergence-free projection of the discrete velocity field, and of its complement:

Figure 5 represents the compression curves of the div-free part of the velocity, and of its complement part, when using isotropic divergence-free wavelets and complement wavelets (see (2)).

The curves clearly show that the non div-free part (arising artificially from the spline interpolation), in the velocity decomposition, is in practice negligible. 


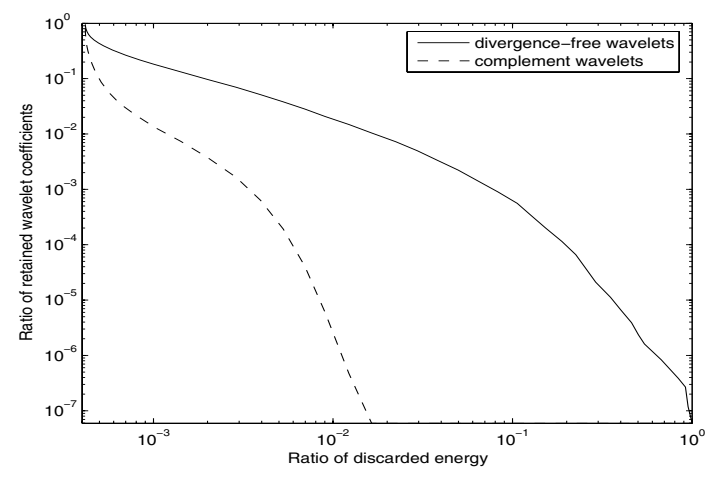

FiguRE 5. Compression error in terms of the number of retained coefficients (log-log scale): div-free part (plain line) and complement part (dashed line) of the velocity field.

\subsection{CVE in the $3 \mathrm{D}$ vorticity field}

We first apply the CVE decomposition with divergence free wavelets to the vorticity field, and we compare our results to those obtained in [15] with orthogonal and biorthogonal bases (respectively Coifman 12 and Harten 3). We use the following notations:

$\omega$ : vorticity field $\left(100 \%\right.$ of the coefficients $\left.N=256^{3}\right)$

$\omega_{\mathrm{c}}$ : vorticity for the coherent part (3\% of the coefficients)

$\omega_{\mathrm{i}}$ : vorticity for the incoherent part (97\% of the coefficients)

$Z=\frac{1}{2}\langle\omega \mid \omega\rangle$ : Enstrophy of the whole field

$$
\omega=\omega_{\mathrm{c}}+\omega_{\mathrm{i}}
$$

$Z_{\mathrm{c}}=\frac{1}{2}<\omega_{\mathrm{c}} \mid \omega_{\mathrm{c}}>$ : Enstrophy of the coherent part

$Z_{\mathrm{i}}=\frac{1}{2}<\omega_{\mathrm{i}}\left|\omega_{\mathrm{i}}\right\rangle:$ Enstrophy of the incoherent part

$<\omega_{\mathrm{c}} \mid \omega_{\mathrm{i}}>$ : cross-term.

Following (5), the cross-term is being computed by

$$
<\omega_{\mathrm{c}} \mid \omega_{\mathrm{i}}>=Z-Z_{\mathrm{c}}-Z_{\mathrm{i}}
$$

The initial vorticity field is plotted in Figure 6 . The coherent and incoherent vorticity parts, using either the divergence-free, orthogonal and biorthogonal decomposition are shown on Figure 7.

The coherent part, obtained by retaining only the $3 \%$ largest wavelet modes, is close to the original field, and retains the coherent vortex tubes present in the total vorticity, similarly to the orthogonal and biorthogonal decompositions.

The incoherent part in the div-free decomposition does not exhibit vortex tubes, although some structures can still be observed. In comparison to the incoherent parts obtained with non divergence-free wavelets, this effect is less pronounced for orthogonal wavelets (Fig. 7, middle) and more pronounced for biorthogonal wavelets (Fig. 7, bottom).

Note that the values of the isosurfaces for the incoherent parts have been reduced by a factor 2 .

The statistics of the resulting fields, provided by the divergence-free, orthogonal and biorthogonal wavelet decompositions are reported in Table 1.

The first important criterion is the norm of the incoherent part which measures the distance between the wavelet approximation and the original field. The second important criterion is the cross-term which measures 


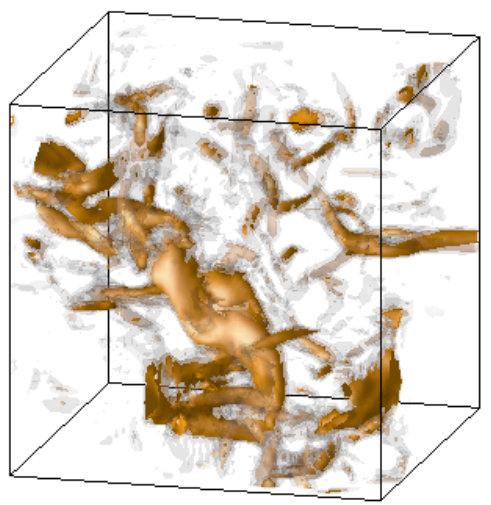

Figure 6. Modulus of the vorticity for the total field. Zoom of the top-left-front-cube of size $64^{3}$. The surfaces, from light to dark, correspond to $\|\vec{\omega}\|=3 \sigma, 4 \sigma$ and $5 \sigma$, with $\sigma=\sqrt{2 Z}$

the correlation between coherent and incoherent part. For practical reasons, the ideal situation is when this cross-term vanishes.

For the three decompositions, only $3 \%$ of the wavelet coefficients retain, for divergence-free biorthogonal wavelets $74.7 \%$ of the enstrophy, for the orthogonal non divergence-free wavelets $75.5 \%$ and for the biorthogonal non divergence-free wavelets $69.0 \%$ of the total enstrophy, while the incoherent parts correspond to $18.1 \%$, $24.4 \%$ and $27.3 \%$, respectively.

In contrast to the orthogonal decomposition, where the cross term vanishes, we observe for both biorthogonal decompositions non vanishing cross terms, i.e. $7.1 \%$ for the divergence-free wavelets and $3.6 \%$ for the non divergence-free wavelets.

As a conclusion for this analysis, the orthogonal wavelets Coifman 12 provide the best results.

\begin{tabular}{|c|cccc|}
\hline $\begin{array}{c}\text { Decomp.field } \\
\text { Vorticity }\end{array}$ & Total & Coherent & Incoherent & Cross-term \\
\hline \%coef & $100 \%$ & $3 \%$ & $97 \%$ \\
\hline & \multicolumn{4}{|c|}{ Divergence-free } \\
Enstrophy & 151.6 & 113.3 & 27.5 & 10.8 \\
\%of Enstrophy & $100 \%$ & $74.7 \%$ & $18.1 \%$ & $7.1 \%$ \\
\hline \hline & \multicolumn{4}{|c}{ Orthogonal } \\
Enstrophy & 151.6 & 114.5 & 0 \\
Enstrophy $(\%)$ & $100 \%$ & $75.5 \%$ & 37.1 & $0 \%$ \\
\hline \hline & 151.6 & Biorthogonal & $5.4 \%$ \\
Enstrophy & 104.6 & 41.4 & $3.6 \%$ \\
Enstrophy $(\%)$ & $100 \%$ & $69.0 \%$ & $27.3 \%$ & \\
\hline
\end{tabular}

TABLE 1. Statistical properties of the vorticity field for the divergence-free, orthogonal-Coifman 12and biorthogonal-Harten 3- decompositions. 
Figure 8 shows the probability distribution function (PDF) of vorticity in semi-log scale, for the divergencefree decomposition. It is to be compared to the ones obtained in [15], and plotted on Figure 9 with orthogonal Coifman-12 (left) and biorthogonal Harten-3 (right) wavelet bases.

The figures show for the three cases that the PDF of the coherent vorticity is very closed to the one of the total vorticity, while the extreme values of the PDFs of the incoherent vorticity are reduced by about a factor three (Fig. 8 and 9, left) and only by a factor two (Fig. 9, right) in the case of biorthogonal non divergence-free wavelets.

Figure 10 shows the isotropic enstrophy spectrum for the total, coherent and incoherent fields for the divergence-free wavelet decomposition. One observes that the coherent spectrum follows the total spectrum in the inertial range, whereas it is steeper in the dissipative range, i.e. for high wavenumbers $(k>30)$. On the other side, the incoherent spectrum corresponds only to wavenumbers $k \geq 30$, namely in the dissipative range.

\subsection{CVE in the $3 \mathrm{D}$ velocity field}

In this section, we apply the CVE using divergence-free wavelets to the velocity field instead of the vorticity field. The CVE method provides a coherent part $\mathbf{u}_{\mathrm{c}}$, and an incoherent part $\mathbf{u}_{\mathrm{i}}$ of the total velocity $\mathbf{u}$. We then compute and plot (Fig. 11) the curl of the coherent and incoherent velocities, that we compare to the coherent and incoherent vorticities (Fig. 7, top) previously computed.

The statistics of the resulting velocity fields are given in Table 2. We compare them to those obtained with orthogonal and non divergence free biorthogonal wavelets, where the coherent and incoherent velocity fields have been computed from the coherent and incoherent vorticity fields previously extracted via the CVE method. In all cases, we observe that only $3 \%$ divergence-free wavelet modes retain about $98.8 \%$ of the total energy, while the remaining $97 \%$ modes contain $0.4 \%$ of the energy. For the non divergence-free decompositions we find in the orthogonal and biorthogonal case that $99.0 \%$ and $98.6 \%$ of the energy are retained by the coherent velocities, while $0.6 \%$ and $0.7 \%$ of the energy are retained by the incoherent velocities, respectively. The crossterms contain $0.8 \%, 0.4 \%$ and $0.7 \%$ of the energy, respectively. Note that the orthogonal decomposition is only orthogonal for vorticity and not for velocity, as wavelets are not eigenfunctions of the Biot-Savart operator used to compute the corresponding velocities from the decomposed vorticities.

\begin{tabular}{|c|cccc|}
\hline $\begin{array}{c}\text { Decomp.field } \\
\text { Velocity }\end{array}$ & Total & Coherent & Incoherent & Cross-term \\
\hline \%coef & $100 \%$ & $3 \%$ & $97 \%$ \\
\hline & \multicolumn{4}{|c|}{ Divergence-free } \\
Energy & 1.358 & 1.342 & 0.006 & 0.010 \\
\%of Energy & $100 \%$ & $98.8 \%$ & $0.4 \%$ & $0.8 \%$ \\
\hline \hline Decomp.field & Total & Coherent & Incoherent & Cross-term \\
Vorticity & \multicolumn{5}{|c}{0.008} \\
\hline Energy & 1.358 & Orthogonal & 0.006 \\
Energy $(\%)$ & $100 \%$ & Biorthogonal \\
\hline & 1.344 & $0.6 \%$ & 0.010 \\
\hline Energy & 1.358 & 1.338 & 0.010 \\
Energy $(\%)$ & $100 \%$ & $98.6 \%$ & $0.7 \%$ & $0.7 \%$ \\
\hline
\end{tabular}

TABLE 2. Statistical properties of the velocity field for the divergence-free decomposition compared to statistical properties of the energy issued from the CVE of the vorticity field with orthogonal and biorthogonal wavelet thresholding. 
Figure 12 shows the PDF of the velocity in semi-log scale, for the divergence-free decomposition, whereas Figure 13 shows the PDF of the velocity, reconstructed from the CVE of the velocity fields (total, coherent and incoherent), in the orthogonal (left) and biorthogonal (right) decomposition.

The curves obtained in the divergence-free case for a CVE on the velocity are very closed to the ones obtained in the orthogonal case with a CVE on the vorticity. In the div-free case, the coherent velocity has the same Gaussian distribution as the total velocity, and the PDF of the incoherent velocity is also almost a Gaussian. This behaviour can be explained by the fact that the coherent velocity and the incoherent velocity are almost orthogonal in the divergence-free decomposition (the cross-term represents only $0.8 \%$ of the total energy), which is not really the case for the coherent and incoherent vorticities in previous section (where the cross-term represents about $7 \%$ of the total enstrophy).

Figure 14 shows the energy spectra associated to the CVE of the velocity field in the divergence-free wavelet decomposition: as one can see, the energy spectrum of the coherent velocity is identical to that of the total velocity along the inertial range, whereas it differs for high wavenumbers corresponding to the dissipative range. For the incoherent flows, the slope of the spectrum is very closed to $k^{2}$, meaning that the velocity is decorrelated in physical space. By comparison, Figure 15 represents the energy spectra associated to the CVE of the vorticity field, with the orthogonal (left) and biorthogonal (right) decompositions. The main difference lies near the Nyquist frequency where the coherent velocity in Figure 14 saturates, instead of decreasing.

As a conclusion, results with the three types of decomposition are comparable. But we can notice here that, although the complexity of the three methods are equivalent, divergence-free wavelets allow to apply the CVE method directly in the velocity field.

\section{CONCLUSiOn}

In the present paper we investigated the interest of divergence-free biorthogonal wavelets for extracting coherent vortices out of turbulent flows. We applied the coherent vortex extraction algorithm based on a nonlinear thresholding of the wavelet coefficients to DNS data of homogeneous isotropic turbulence at $R_{\lambda}=150$. In the first part we applied the algorithm to the vorticity field. We found that the divergence-free biorthogonal wavelets yield similar results than orthogonal wavelets, which are better than those with biorthogonal non divergence-free wavelets. In the second part we applied the coherent vortex extraction algorithm to the velocity field using divergence-free biorthogonal wavelets. We observed that the results are comparable to those with orthogonal and non divergence free biorthogonal wavelets, although in these two last cases the coherent and incoherent velocity parts are computed from the coherent and incoherent vorticity fields previously extracted. The obtained results motivate the use of divergence-free wavelets for Coherent Vortex Simulation [9, 16], where the time evolution of the coherent flow is deterministically computed in an adaptive wavelet basis.

The authors thank the CIRM Marseille for its hospitality and for financial support during the CEMRACS 2005 summer-program where part of the work was carried out. We also thankfully acknowledge financial support from the European Union project IHP on 'Breaking Complexity', contract HPRN-CT-2002-00286.

\section{REFERENCES}

[1] C.-M. Albukrek, K. Urban, D. Rempfer, and J.-L. Lumley, Divergence-Free Wavelet Analysis of Turbulent Flows, J. of Scientific Computing 17(1): 49-66, 2002.

[2] A. Azzalini, M. Farge and K. Schneider.

Nonlinear wavelet thresholding: A recursive method to determine the optimal denoising threshold.

Appl. Comput. Harm. Anal., 18(2), 177-185, 2005.

[3] M. O. Domingues, I. Broemstrup, K. Schneider, M. Farge, B. Kadoch Coherent Vortex Extraction in 3D homogeneous isotropic turbulence using orthogonal wavelets, 
In this issue.

[4] A. Cohen, I. Daubechies and J.C. Feauveau,

Biorthogonal bases of compactly supported wavelets,

Comm. Pure Appl. Math., 45, 485-560, 1992.

[5] E. Deriaz,

Ondelettes pour la simulation des écoulements fluides incompressibles en turbulence.

Thèse de Doctorat, Institut National Polytechnique de Grenoble, mars 2006.

[6] E. Deriaz and V. Perrier,

Divergence-free Wavelets in 2D and 3D, application to the Navier-Stokes equations.

J. of Turbulence, 7(3), 1-37, 2006.

[7] D. Donoho and I. Johnstone.

Ideal spatial adaptation via wavelet shrinkage.

Biometrika, 81:425-455, 1994.

[8] M. Farge.

Wavelet transforms and their applications to turbulence.

Ann. Rev. Fluid Mech., 24:395, 1992.

[9] M. Farge, K. Schneider and N. Kevlahan.

Non-Gaussianity and Coherent Vortex Simulation for

two-dimensional turbulence using an adaptive orthonormal wavelet

basis.

Phys. Fluids, 11(8), 2187-2201, 1999.

[10] M. Farge, G. Pellegrino and K. Schneider.

Coherent vortex extraction in 3D turbulent flows using orthogonal wavelets.

Phys. Rev. Lett., 87(5), 45011-45014, 2001.

[11] M. Farge, K. Schneider, G. Pellegrino, A. Wray and B. Rogallo.

Coherent vortex extraction in three-dimensional homogeneous turbulence:

Comparison between CVS-wavelet and POD-Fourier decompositions.

Phys. Fluids, 15(10), 2886-2896, 2003.

[12] J.-P. Kahane and P.G. Lemarié-Rieusset, Fourier series and wavelets, book, Gordon \& Breach, London, 1995.

[13] P.G. Lemarié-Rieusset, Analyses multi-résolutions non orthogonales, commutation entre projecteurs et dérivation et ondelettes vecteurs à divergence nulle (in french),

Revista Matemática Iberoamericana, 8(2): 221-236, 1992.

[14] S. Mallat,

A Wavelet Tour of Signal Processing,

Academic Press, 1998.

[15] O. Roussel, K. Schneider and M. Farge.

Coherent vortex extraction in 3D homogeneous turbulence:

comparison between orthogonal and biorthogonal wavelet decompositions.

J. of Turbulence, 6(11):1-15, 2005.

[16] K. Schneider, M. Farge, G. Pellegrino, and M. Rogers.

Coherent Vortex Simulation of 3D turbulent mixing layers using orthogonal wavelets.

J. Fluid Mech., 534, 39-66, 2005.

[17] K. Urban, Using divergence-free wavelets for the numerical solution of the Stokes problem, AMLI'96: Proceedings of the Conference on

Algebraic Multilevel Iteration Methods with Applications,

2: 261-277, University of Nijmegen, The Netherlands, 1996.

[18] K. Urban, Wavelet Bases in $H$ (div) and H(curl), Mathematics of Computation 70(234): 739-766, 2000.

[19] K. Urban, Wavelets in Numerical Simulation, Springer, 2002.

[20] A. Vincent, M. Meneguzzi,

The spatial structure and statistical properties of homogeneous turbulence,

Journal of Fluid Mechanics 225, 1-20, 1991. 

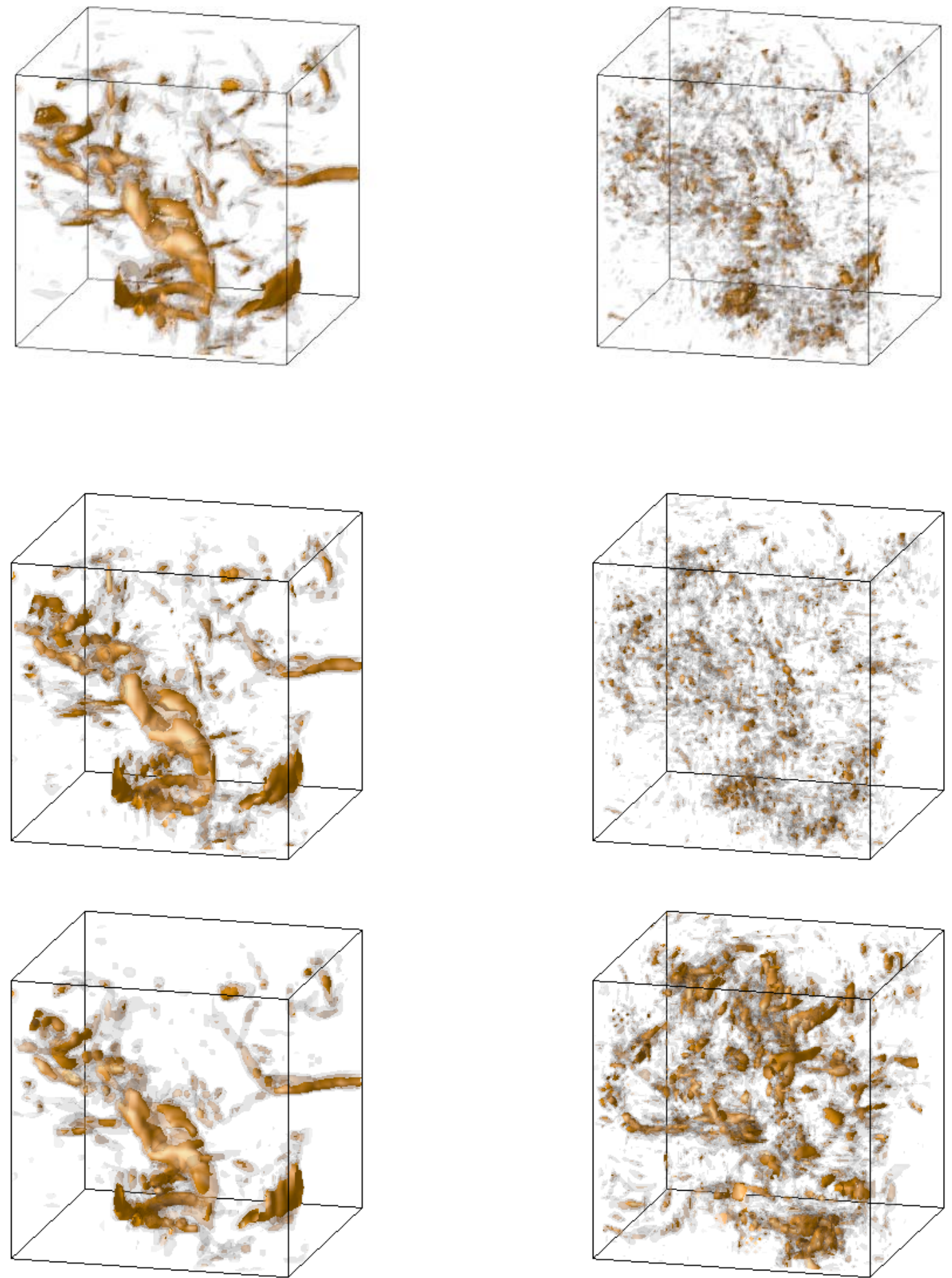

FiguRE 7. Comparison between divergence-free biorthogonal wavelets (top) and non divergence free orthogonal (middle) or biorthogonal (bottom) wavelets. Modulus of the vorticity for the coherent part (left) and incoherent part (right) of the CVE method. Zoom of a cube of size $64^{3}$ (from the second row, second line and second column). The isosurfaces, from light to dark, correspond to $\|\vec{\omega}\|=3 \sigma, 4 \sigma$ and $5 \sigma$ on the left side, $\|\vec{\omega}\|=\frac{3}{2} \sigma, 2 \sigma$ and $\frac{5}{2} \sigma$ on the right side. 


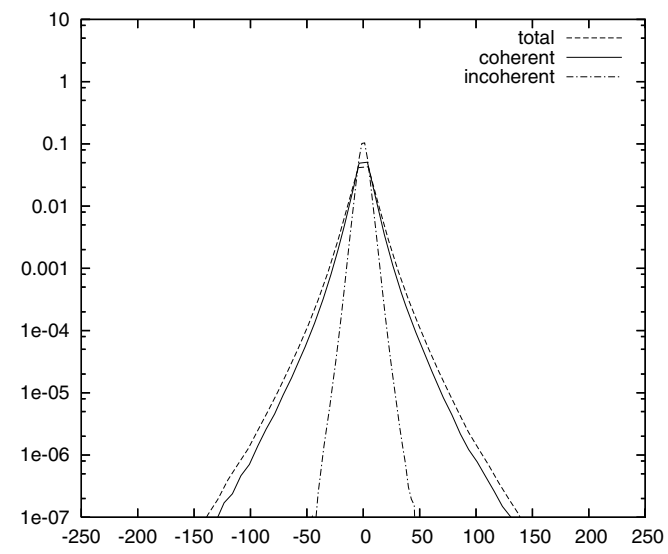

FIGURE 8. PDF (probability distribution function) of vorticity associated to the divergence-free biorthogonal wavelet decomposition.
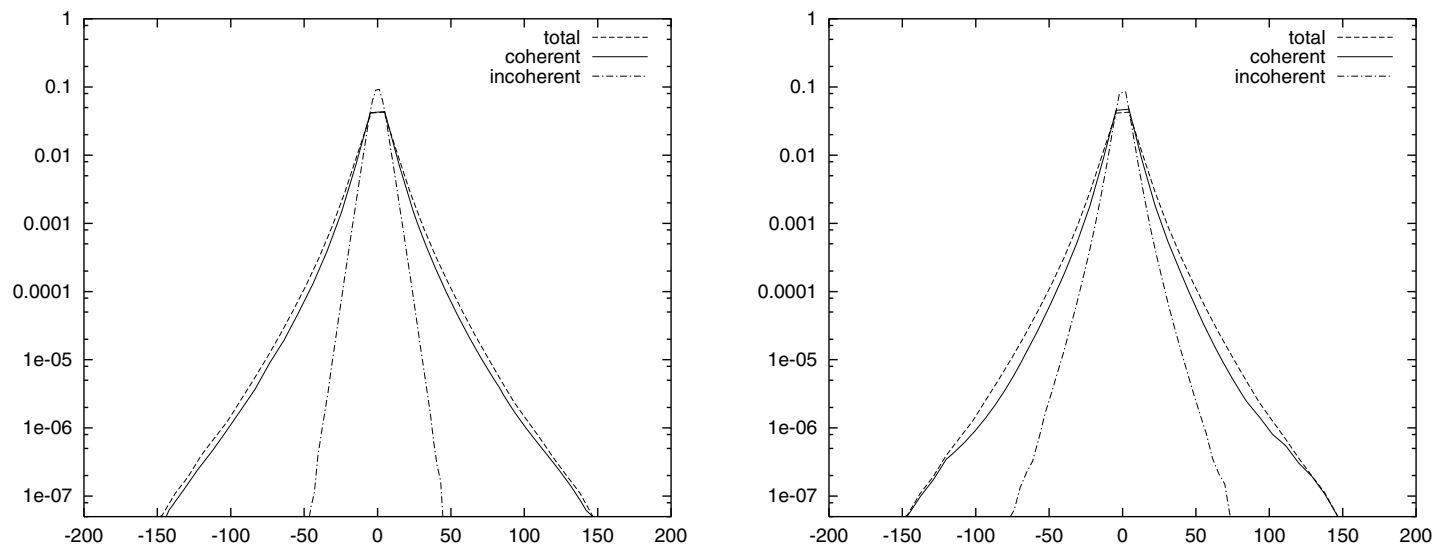

FIGURE 9. PDF (probability distribution function) of vorticity associated to the orthogonal (left) and biorthogonal (right) non divergence-free wavelet decomposition. 


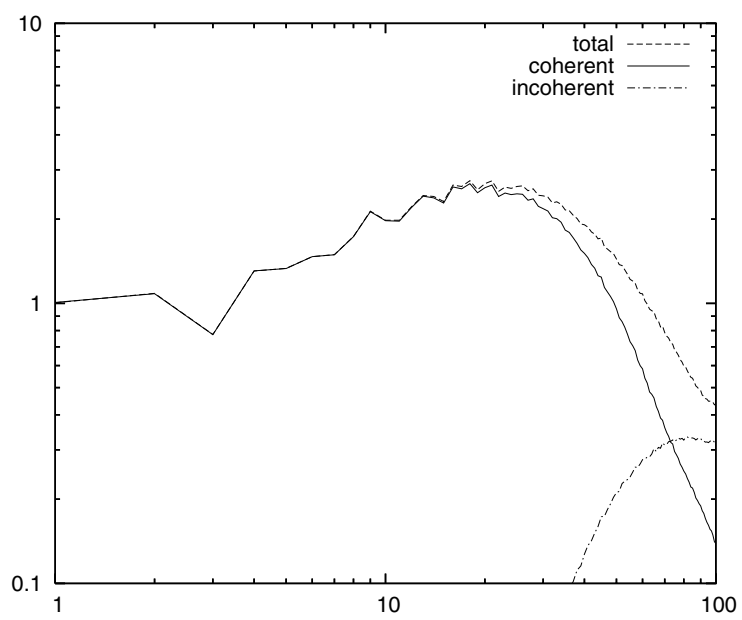

FIGURE 10. Enstrophy spectra obtained by divergence-free wavelet decomposition. x-axis: wavenumber, y-axis: enstrophy spectrum.
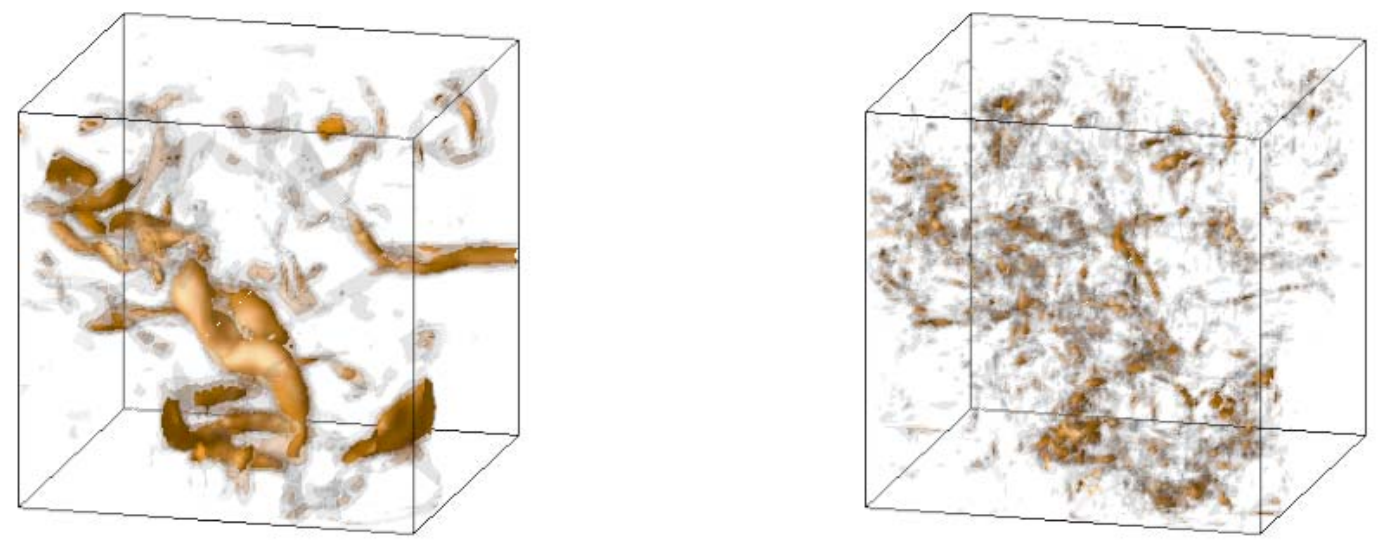

FIGURE 11. Divergence-free wavelet decomposition. Modulus of the vorticity field associated to the coherent velocity (left) and the vorticity field associated the incoherent velocity (right) of the CVE method. Zoom of the top-left-front-cube of size $64^{3}$. The surfaces, from light to dark, correspond to $\|\vec{\omega}\|=3 \sigma, 4 \sigma$ and $5 \sigma$ on the left side, $\|\vec{\omega}\|=\frac{3}{2} \sigma, 2 \sigma$ and $\frac{5}{2} \sigma$ on the right side. 


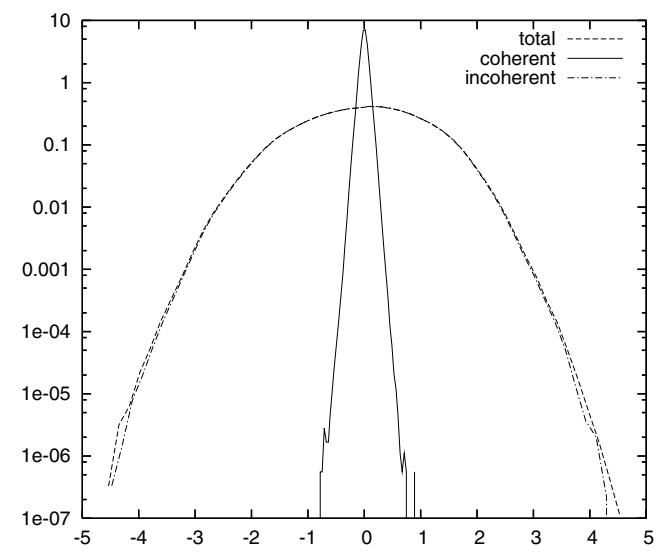

FIGURE 12. PDF (probability distribution function) of velocity associated to the divergence-free wavelet compression with $3 \%$ of the coefficients.
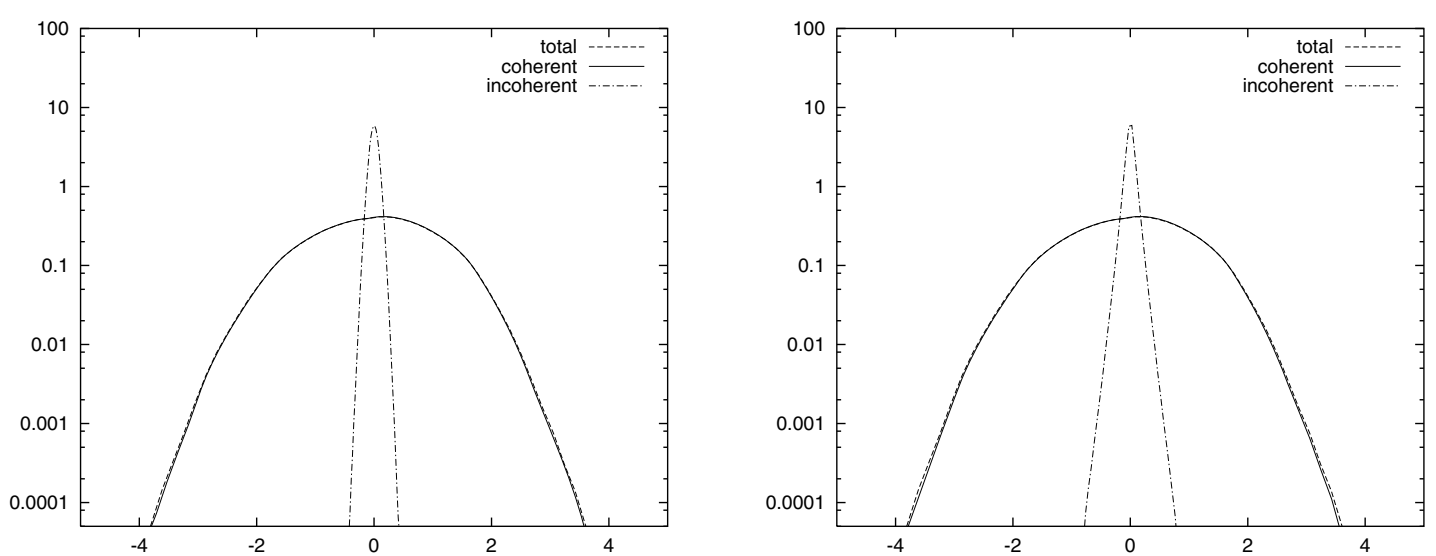

FIGURE 13. PDF (probability distribution function) of velocity associated to the orthogonal (left) and biorthogonal (right) wavelet decomposition. 


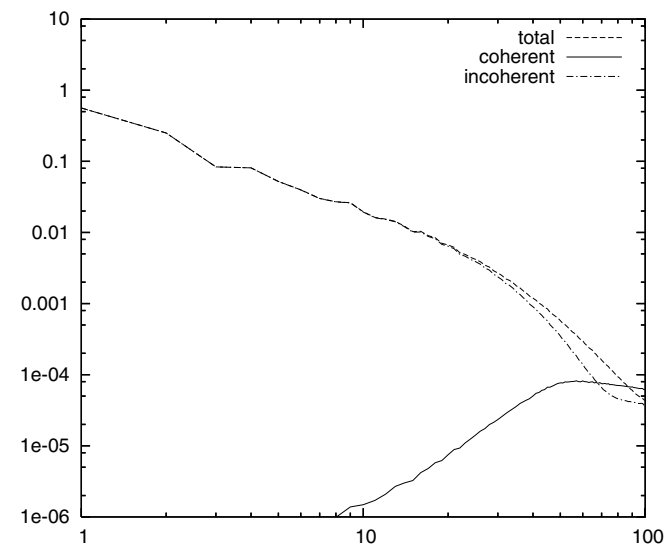

FIGURE 14. Energy spectra associated to the CVE of the vorticity field: divergence-free wavelets. -axis: wavenumber, y-axis: energy spectrum.
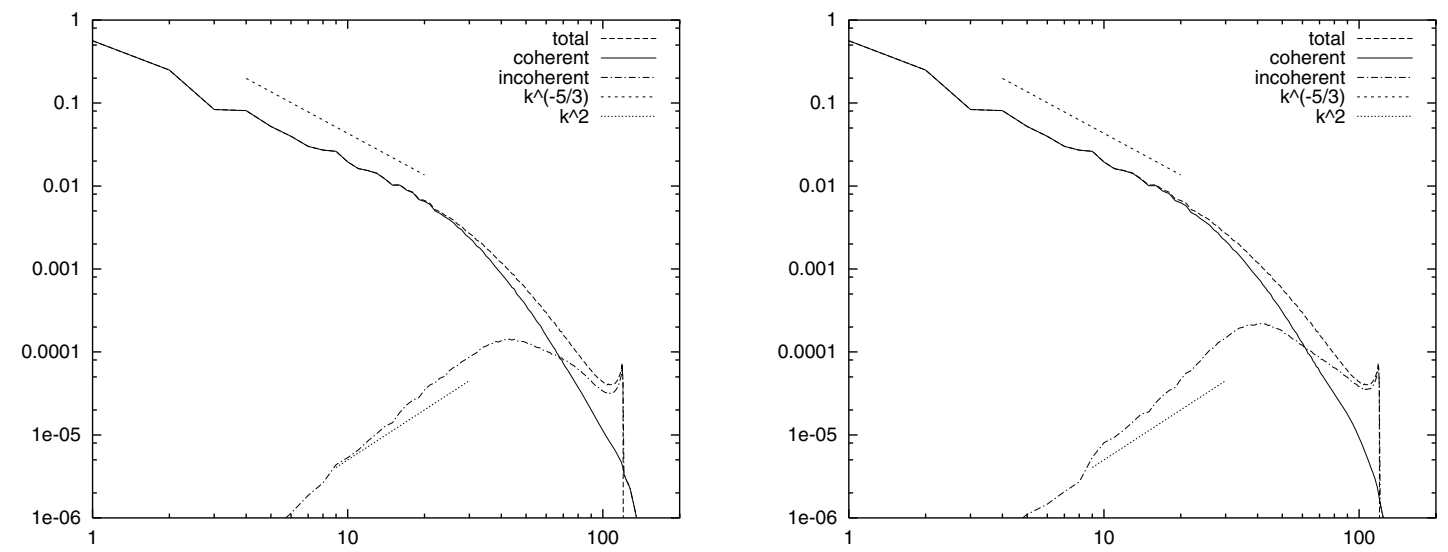

FIGURE 15. Energy spectra associated to the CVE of the vorticity field: orthogonal (left) and biorthogonal (right) wavelet decomposition. x-axis: wavenumber, y-axis: energy spectrum. 